\title{
An Evolution Mechanism for Dynamic Physical Applications in the Internet of Things
}

\author{
Kaibin Xie ${ }^{1,2}$, Haiming Chen ${ }^{1}$, Dong $\mathrm{Li}^{1}$ and $\mathrm{Li} \mathrm{Cui}^{1}$ \\ ${ }^{1}$ Institute of Computing Technology, Chinese Academy of Sciences, Beijing \\ ${ }^{2}$ University of Chinese Academy of Sciences, Beijing \\ Email: xiekaibin@ict.ac.cn, chenhaiming@ict.ac.cn, lidong@ict.ac.cn and lcui@ict.ac.cn
}

\begin{abstract}
With rapid development of the Internet of Things, more and more smart devices are deployed in the physical space. A physical application is composed by several smart devices which provide physical data. The physical applications need appropriate physical information processing systems to process the related data. However, the physical applications are dynamic because of the ever-changing demands in the IoT. So it is necessary to design an evolution mechanism for the dynamic physical applications to find appropriate physical information processing systems. We first analyze the changing types of dynamic physical applications. Then we conclude three relationships between the dynamic physical applications and physical information processing systems. In order to verify the correctness of the evolution mechanism, we use Communication Sequential Process to formalize the evolution mechanism and use Process Analysis Toolkit to verify deadlock-free, divergence-free and nonterminating of the evolution mechanism.
\end{abstract}

Keywords-Internet of Things; physical application; physical information processing system; dynamic; evolution mechanism

\section{INTRODUCTION}

The concept of the Internet of Things (IoT) is proposed by MIT in 1999 [6] and has got extensive attention from the industrial community [3]. According to the vision for the IoT [7], most smart devices in the physical space have the ability to communicate and compute. A physical application is composed by several smart devices which provide physical data to the social. These physical applications need appropriate physical information processing systems to process the related data.

In our previous work, we have established a software architecture of the IoT, named PMDA [9]. The PMDA is composed by three models which are extracted from the social space, the virtual space and the physical space. The three models are the Application Model, the Sense-Execute Model and the Physical Model. The relationship of the three models is illustrated in Fig. 1. As depicted in Fig. 1, the Application Model sends requirement information (req-info) to the SenseExecute Model; the Sense-Execute Model processes sensory data (sen-data) from the Physical Model according to the reqinfo from the Application Model and sends the execution information (exe-info) to control the Physical Model; the Physical Model provides sen-data to the Sense-Execute Model and receives the exe-info from the Sense-Execute Model.

According to the PMDA, we can see that these physical applications can be regard as the instances of the Physical Model; the physical information processing systems can be regard as the instances of the Sense-Execute Model.
Because of the ever-changing demands in the IoT [10], the physical space and the physical parameters of physical applications are changeable. The changeable physical applications need appropriate information processing systems. In order to adapt the ever-changing demands in the IoT, we design an evolution mechanism for the dynamic physical applications to find appropriate physical information processing systems.

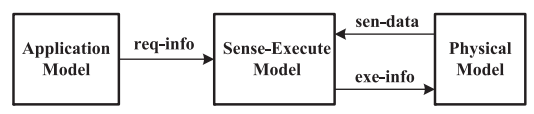

Fig. 1. The relationship of the three models in the PMDA

The challenges for designing the evolution mechanism are as follows. Firstly, it is difficult to find an appropriate physical information processing system for a dynamic physical application in the IoT. Secondly, it is hard to illustrate the correctness of the evolution mechanism. Without strict proof, we can not state that the evolution mechanism is correct in all situations.

The remainder of this article is organized as follows. We provide the related work and a motivational scenario of the evolution mechanism in Section II. Section III analyzes the dynamic changes of physical applications. Three relationships between the dynamic changes of these physical applications and the physical information processing systems are depicted in Section IV. Section V establishes the procedures of the evolution mechanism. The processes of evolution mechanism are depicted by the Communication Sequential Process(CSP) [5] statements and the correctness of the evolution mechanism is verified by the Process Analysis Toolkit(PAT) [4] in Section VI. Finally, we make a concluding remark of the evolution mechanism.

\section{RELATED WORK AND MOTIVATIONAL SCENARIO}

The evolution for the IoT has been investigated mainly in three aspects which are the changing context and demands for an IoT application [2], the user mobility and unreliable sensor availability in IoT [1] and the dynamic interactions in the IoT [8].

Based on the software architecture PMDA and recent research in IoT evolution, this article analyzes the evolution mechanism for the dynamically changing physical applications due to the ever-changing demands in the IoT. The evolution mechanism can guarantee that these physical applications 
evolve correctly according to the physical information processing systems in the IoT. We depict a typical scenario for these dynamic physical applications as follows.

Jim is a supervisor of a large environment monitoring system. The organization deploys environmental monitoring applications in three areas (area A, area B and area C) of a city. Jim deploys the sensors in the three areas in order to provide physical data of the environment to the corresponding physical information processing systems which can process the physical data according to the requirements (Req-A, Req$B$ and Req-C) from the social space. In area A, Jim deploys temperature sensors and humidity sensors; in area B, Jim deploys $\mathrm{CO}$ sensors and $\mathrm{CO}_{2}$ sensors; in area $\mathrm{C}$, Jim deploys $\mathrm{CO}$ sensors and temperature sensors. The three environmental monitoring applications are instances of the Application Model and named as pma, pmb and pmc respectively. Jim develops three physical information processing systems to process the three environmental monitoring applications. The three physical information processing systems are instances of the Sense-Execute Model and named as sema, semb and semc respectively. Fig. 2 shows the scenario of the three environmental monitoring applications.

Jim wants to manage the city's environmental monitoring applications in an effective way even when the environmental monitoring applications have changed. But the above environmental monitoring applications don't conform to Jim's expectations because the three physical information processing systems can not adapt to the dynamic changes in the three environmental monitoring applications.

So Jim asks the Research department to realize the intended environmental monitoring applications. The Research department reports that they should design an evolution mechanism for these environmental monitoring applications. The evolution mechanism can adapt to the dynamic changes in the three environmental monitoring applications and guarantee that there are appropriate physical information processing systems for the three environmental monitoring applications.

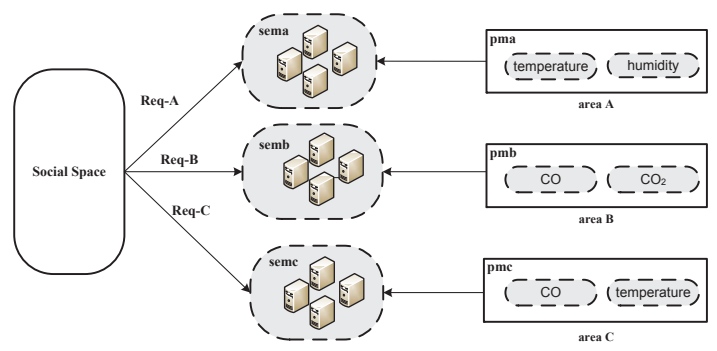

Fig. 2. The scenario of the three environmental monitoring applications

\section{CHANGING TYPES OF PHYSICAL APPLICATIONS}

Physical location and the physical parameters are two key characteristics for the physical applications. The structure of a physical application is illustrated in Fig. 3. In Fig. 3, the pa represents the name of a physical application; the pl represents the physical location of a physical application and the pps represents the physical parameters of a physical application.

\begin{tabular}{|l|l|}
\hline \multicolumn{2}{|l|}{ semn=moniABCD } \\
\hline pro $=\mathrm{CO}+\mathrm{CO}_{2}+\mathrm{pH}+\mathrm{O}_{2}$ \\
\hline $\mathrm{pl}=\mathrm{A}$ & $\mathrm{pps}=\mathrm{CO}+\mathrm{pH}$ \\
\hline$\ldots$ & $\ldots$ \\
\hline $\mathrm{pl}=\mathrm{D}$ & $\mathrm{pps}=\mathrm{O}_{2}$ \\
\hline
\end{tabular}

Fig. 3. The structure of a physical Fig. 4. The structure of a physical application information processing system

We take the scenario in section II as an example and the possible changing types of the dynamic physical applications are as follows.

\section{A. Changing types for the $p l$}

There are four changing types which are changed in the $\mathrm{pl}$ and remain unchanged in the pps. The four changing types can be depicted as follows.

1) Shrink the scope of the physical location in the physical application. The reduced sites are denoted by $\mathrm{S}$. We use SHRINK to represent this changing type.

2) Enlarge the scope of the physical location in the physical application. The added sites are denoted by E. We use ENLARGE to represent this changing type.

3) Shrink and then enlarge the scope of the physical location in the physical application or the versa. We use SHR-ENL to represent this changing type.

4) Move to a new physical location. We use MOVE to represent this changing type.

\section{B. Changing types for the pps}

There are four changing types for the pps, which are changed in the pps and remain unchanged in the pl.

1) Add new physical parameters. We use ADD to represent this changing type.

2) Delete physical parameters. We use DELETE to represent this changing type.

3) Delete the physical parameters and then add new physical parameters or the versa. We use DEL-ADD to represent this changing type.

4) New physical parameters. We use NEW to represent this changing type.

\section{Changing types for both the pl and the pps}

Because there are four changing types for the $\mathrm{pl}$ and four changing types for the pps, we can conclude that there are sixteen changing types for the changes in both the $\mathrm{pl}$ and the pps of a physical application.

\section{ZERO changing type}

There is a special changing type for the physical application, which denotes that the $\mathrm{pl}$ or the pps of a physical application is null. We use ZERO to represent this changing type. The ZERO denotes that the physical application has terminated in the IoT. 


\section{RELATIONSHIPS BETWEEN DYNAMIC PHYSICAL APPLICATIONS AND PHYSICAL INFORMATION PROCESSING SYSTEMS}

According to these changing types discussed in section III, we analyze the relationships between the dynamic physical applications and the physical information processing systems.

A physical information processing system processes the physical data from the corresponding physical applications according to the requirements from the social space. The process ability of a physical information processing system is denoted by pro. The pro is composed by several physical parameters which the physical information processing system can process in the IoT. A physical information processing system can process these physical applications if the pps of these physical applications are contained by the pro. A physical information processing system records the related $\mathrm{pl}$ and $\mathrm{pps}$ of the physical applications. We use semn to represent the name of a physical information processing system.

The structure of a physical information processing system is illustrated in Fig. 4.

We conclude that there are three relationships which are named as ERASE, UPDATE and LOOKUP and analyze the three relationships as follows.

\section{A. The ERASE relationship}

The ERASE relationship denotes that the changed physical application has nothing with any physical information processing systems in the IoT. We can conclude that the physical application has terminated in the IoT. The changing type for the ERASE relationship is ZERO.

\section{B. The UPDATE relationship}

If a physical application has changed and the changed pps for the physical application still contains in the pro of the corresponding physical information processing system, we name it as UPDATE relationship for the physical application.

\section{The LOOKUP relationship}

If a physical application has changed and the changed pps for the physical application is not in the pro of the corresponding physical processing system, we name it as LOOKUP relationship for the physical application.

\section{EVOLUTION PROCEDURES}

According to the procedures of the three relationships, we can conclude that there are seven procedures for the evolution mechanism. We illustrate the seven procedures as follows.

1) IRS (Initial Relationship Set): The IRS denotes the relationship between the physical applications and the physical information processing systems at initial.

2) JUDGE: We judge the changing types for these dynamic physical applications in the IoT.

3) ERASE: The relationship is ERASE. After the procedure for ERASE, goto 7).

4) UPDATE: The relationship is UPDATE. After the procedure for UPDATE, goto 7)
5) LOOKUP: The relationship is LOOKUP. If we can find an appropriate physical information processing system for the changed physical application, goto 7). Else, it denotes that there is no physical information processing system for the changed physical application, goto 6 )

6) DEPLOY: We deploy a new physical information processing system for the changed physical application and establish the relationship between the changed physical application and the new physical information processing system. After the procedure for DEPLOY, goto 7).

7) UPDIRS: We update the IRS in order to form a new relationship between the changed physical applications and the physical information processing systems. After the procedure for UPDIRS, goto 1).

The seven procedures of the evolution mechanism are illustrated in Fig. 5.

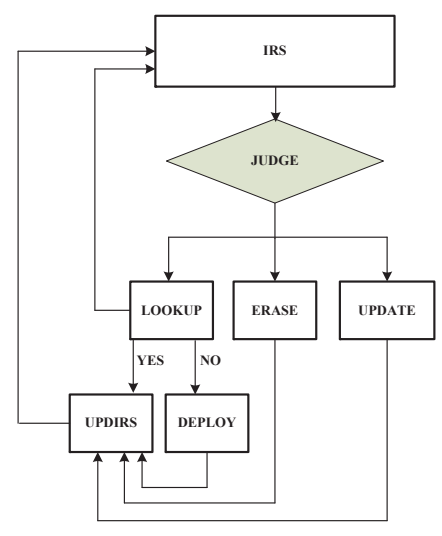

Fig. 5. Procedures of the evolution mechanism

\section{CORRECTNESS VERIFICATION}

In order to verify the correctness of the procedures for the evolution mechanism, we express the seven procedures by the processes in the CSP. We verify the correctness of these processes by the PAT.

\section{A. Processes for the evolution mechanism}

We use the process IRS to express the procedure 1) in the evolution mechanism. There is only one event (generate) in the IRS. The meaning of the event is to generate the Initial Relationship Set.

We use the process JUDGE to express the procedure 2). There are two events (change and judge) in the process JUDGE. Event "change"shows that the physical application has changed. Event "judge"is to judge the relationship.

We use process ERASE to express the procedure 3). There are two events (erase, unlinkera) in the process ERASE. Event "erase"shows that the physical information processing system deletes the related pl and pps of the physical application. Event "unlinkera"shows that the physical information processing system unlinks with the physical application. 
We use the process UPDATE to express the procedure 4). There is only one event (update) in the process UPDATE. Event "update"shows that the physical information processing system updates the pl and the pps.

We use process LOOKUP to express the procedure 5). There are three events (delup, unlinkup and search) in the process UPDATE. Event "delup"shows that the physical information processing system deletes the pl and the pps of the related physical application. Event "unlinkup"shows that the physical information processing system unlinks with the physical application. Event "search"shows that the physical application searches the appropriate physical information processing system in the IoT.

We use process DEPLOY to express the procedure 6). There are two events (link, register) in the process DEPLOY. Event "link"shows that the physical application links to a new physical information processing system. Event "register"shows that the $\mathrm{pl}$ and pps of the physical application are registered in the new physical information processing system.

We use four processes (ERAIRS, UPDIRS, LOOKIRS and DEPIRS) to express the procedure 7).

The process ERAIRS updates the IRS in procedure 7) after the process ERASE. The process UPDIRS updates the IRS in procedure 7) after the process UPDATE. The process LOOKIRS updates the IRS in procedure 7) after the process LOOKUP. The process DEPIRS updates the IRS in procedure 7) after the process DEPLOY.

There is only one event "updera"in the process ERAIRS. Event "updera"updates the IRS by deleting the link relationship.

There is only one event "updup"in the process UPDIRS. Event "updup"updates the IRS by updating the pl and pps of the physical application in the physical information processing system.

There is only one event "updlook"in the process LOOKIRS. Event "updlook"updates the IRS by adding the pl and pps of the physical application to the physical information processing system.

There is only one event "upddep"in the process DEPIRS. Event "upddep"updates the IRS by adding the pl and pps of the physical application to the new physical information processing system.

Based on the above analysis of the processes and events we can get ten CSP processes for the procedures of the evolution mechanism as follows.

- $\quad I R S=$ generate $\rightarrow J U D G E ;$

- $J U D G E \rightarrow$ change $\rightarrow$ judge $\rightarrow$ (ERASE[*]UPDATE[*]LOOKUP);

- $\quad E R A S E=$ erase $\rightarrow$ unlinkera $\rightarrow$ ERAIRS

- $\quad U P D A T E=$ update $\rightarrow U P D I R S$;

- $L O O K U P=$ delup $\rightarrow$ unlinkup $\rightarrow$ search $\rightarrow$ (DEPLOY [*]LOOKIRS);

- DEPLOY $=$ link $\rightarrow$ register $\rightarrow$ DEPIRS;
- $\quad E R A I R S=$ updera $\rightarrow I R S$

- $U P D I R S=u p d u p \rightarrow I R S$;

- $L O O K I R S=$ updlook $\rightarrow I R S$

- $D E P I R S=$ upddep $\rightarrow I R S$

\section{B. Verification results for the evolution mechanism}

We use process EM to express the behavior of the whole procedures in the evolution mechanism. Because the process IRS can be regard as the first process in the evolution mechanism, the process IRS is equal to the process EM.

Based on the PAT, we can verify that the process EM is deadlock-free, divergence-free and nonterminating. The results for the processes of the evolution mechanism are illustrated in Fig. 6.

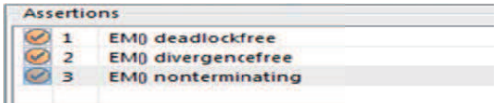

Fig. 6. The results for the processes of the evolution mechanism

\section{CONCLUSION}

This paper provides a novel evolution mechanism between the dynamic physical applications and the corresponding physical information processing systems in the IoT. The evolution mechanism satisfies three properties which are deadlock-free, divergence-free and nonterminating.

\section{ACKNOWLEDGMENT}

Partial work of this paper is supported by the International S\&T Cooperation Program of China (ISTCP) under Grant No.2013DFA10690.

\section{REFERENCES}

[1] R. Arun, P. Davy and B. Yolande. Enabling self-learning in dynamic and open IoT environments. Procedia Computer Science, pp.207-214, 2014.

[2] A.P. Athreya, B. DeBruhl and P. Tague. Designing for self-configuration and self-adaptation in the Internet of Things. IEEE International Conference Conference on Collaborative Computing: Networking, Applications and Worksharing (Collaboratecom), 2013.

[3] Y. Cheng, X. Li, Z. Li, et al. AirCloud: a cloud-based air-quality monitoring system for everyone. $12^{\text {th }}$ ACM Conference on Embedded Network Sensor Systems (SenSys), pp.251-265, 2014.

[4] CS Department NUS. PAT: Process Analysis Toolkit. Available: http://www. patroot. com.

[5] C.A.R. Hoare. Communicating Sequential Processes. New Jersey: Prentice-Hall International, 1985.

[6] G.Neil and C. Danny. Internet 0: Interdevice internetworking-end-toend modulation for embedded networks. IEEE Circuits and Devices Magazine, pp.48-55, 2006.

[7] OECD. Machine-to-machine communications: Connecting billions of devices. Available: http://www.oecdilibrary. org, 2012.

[8] L. Rao, C. Fan, Y. Wu, et al. A Self-Adapting Dynamic Service Management Platform for Internet of Things. LISS 2013, Springer Berlin Heidelberg, pp.783-791, 2015.

[9] K. Xie, H. Chen and L. Cui. PMDA: A physical model driven software architecture for Internet of Things. Computer Research and Development, pp.1185-1197, 2013(In Chinese).

[10] H. Zhuge. Semantic linking through spaces for cyber-physical-socio intelligence: A methodology. Artificial Intelligence, pp.988-1019, 2011. 